\title{
Industry 4.0 and its applications in orthopaedics
}

\section{Dear Editor,}

Industry 4.0 is an intelligent manufacturing system, which focuses on the design, manufacturing and in providing customized product \& services as per the individual requirements. It encourages the integration of different intelligent manufacturing systems and advanced information technologies and is also known as the fourth revolution. Customization of products is an important issue in orthopaedics and is well taken by the Industry 4.0, as it can make manufacturing systems intelligent \& responsive to customer's need and the delight. ${ }^{1}$ We are contemplating extensive research in this crucial area through different components of Industry 4.0.

Industry 4.0 is anticipated to be operational from 2020 and may realize its extensive capability to fulfill the future needs of the medical and their associated fields. It is highly dependent on innovations like additive manufacturing technologies, process automation, Internet of Things (IoT), artificial intelligence, big data, automation, virtual reality, and robotics. ${ }^{2}$ It is a new phase of development to create new developments in the various medical fields, including orthopaedics. It is supposed to impact the environment extensively by reducing wastage of material and human efforts with the help of various advanced innovative technologies.

Product customization is the desired requirement in orthopaedics because patient-specific implants and devices vary from patient to patient. Industry 4.0 consists of a smart manufacturing system that easily fulfills the customized requirements in orthopaedics. It can provide the capability to create a customized implant to meet the personalized requirement of the patient. It helps through quick design and manufacturing of high-quality implants with different shape \& size, bio-models, surgical tools, and other orthopaedic tools \& devices. Implants manufactured by smart manufacturing technologies comfortably fit the patients. ${ }^{3}$

Industry 4.0 employs artificial intelligence to program machines for difficult situations. Virtual reality provides better information to doctors and patients and is quite useful for simulations and 3D view of the equipment and of the patients. Holography can help in better teaching and training. Thus, Industry 4.0 helps to improve the quality of surgical planning and reduces patient suffering. Table 1 discusses the various clinical applications of Industry 4.0 as envisaged in Orthopaedics.

Smart manufacturing machines and processes used in Industry 4.0 help produce accurate implants with the better finish by using a different variety of materials. These implants need a variety of suitable materials as per the specific patient problem and can also help in complex surgical cases. It can help improve the communication between doctors and the patient. ${ }^{4}$

Industry 4.0 provides direction for the research and development of personalized prostheses and other implants, tools, and

Table 1

Clinical applications of Industry 4.0 in Orthopaedics.

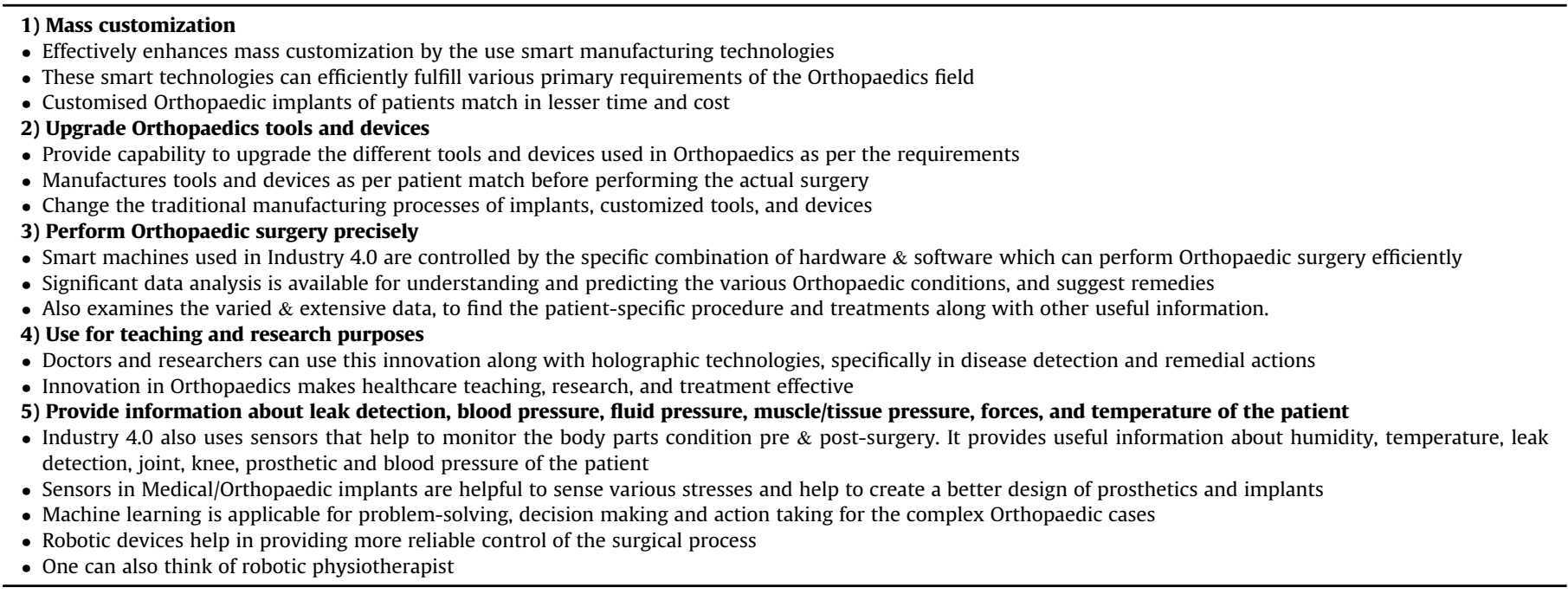


device which can be tested and used for research \& development purpose. There are various unique requirements can be fulfilled by Industry 4.0 in the medical field using virtual reality. ${ }^{5}$

In the upcoming years, Industry 4.0 is to bring innovations to the field of Orthopaedics. By Industry 4.0 applications, medical students can be adequately trained to detect problems and correlate them. With these smart manufacturing technologies of Industry 4.0, data collected from different patients can be analyzed adequately for disease detection, control, and future remedial action. In future, Industry 4.0 will adapt to the world-class manufacturing system that efficiently creates smarter medical as well as orthopaedics products.

\section{Conflicts of interest}

None.

\section{Appendix A. Supplementary data}

Supplementary data to this article can be found online at https://doi.org/10.1016/j.jcot.2018.09.015.

\section{References}

1. Vaishya R, Vijay V, Agarwal AK. Total knee arthroplasty using patient-specific blocks after prior femoral fracture without hardware removal. Indian J Orthop.
2018;52(2):154-160

2. Liao Y, Deschamps F, LouresEDFR, Ramos, LFP. Past, present, and future of Industry 4.0-A systematic literature review and research agenda proposal. Int J Prod Res. 2017;55(12):3609-3629.

3. Pace P, Aloi G, Gravina R, Caliciuri G, Fortino G, Liotta A. An edge-based architecture to support efficient applications for healthcare Industry 4.0. IEEE Transactions on Industrial Informatics. 2018. https://doi.org/10.1109/TII.2018.2843169.

4. Elhoseny M, Abdelaziz A, Salama AS, Riad AM, Muhammad K, Sangaiah AK. A hybrid model of the Internet of Things and cloud computing to manage big data in health services applications. Future Generat Comput Syst. 2018;86: 1383-1394.

5. Pang Z, Yang G, Khedri R, Zhang YT. Introduction to the special section: convergence of automation technology, biomedical engineering, and health informatics toward the healthcare 4.0. IEEE Reviews in Biomedical Engineering. 2018;11: 249-259.

Abid Haleem, Mohd Javaid*

Department of Mechanical Engineering, Jamia Millia Islamia, New

Delhi, India

Raju Vaishya

Department of Orthopaedics, Indraprastha Apollo Hospital, SaritaVihar, Mathura Road, 110076, New Delhi, India E-mail address: raju.vaishya@gmail.com.

* Corresponding author. E-mail addresses: ahaleem@jmi.ac.in (A. Haleem), mjavaid@jmi.ac.in (M. Javaid).

29 August 2018

Available online 28 September 2018 